\title{
Economic Evaluation of Ticagrelor in Treating Patients with Acute Coronary Syndrome in Hong Kong: A Cost-Utility Analysis
}

\author{
David Bin-Chia Wu ${ }^{1}$, June Wai Yee Choon ${ }^{1}{ }^{*}$, Chun Wie Chong ${ }^{1}$, Benjamin Shing Cheung Lee ${ }^{2}$, \\ Kenneth Kwing Chin Lee ${ }^{1}$
}

${ }^{1}$ School of Pharmacy, Monash University Malaysia, Bandar Sunway, Malaysia

${ }^{2}$ Prince of Wales Hospital, Shatin, Hong Kong

\section{Email address:}

david.wu@monash.edu (D. Bin-Chia Wu), choon.wai.yee@monash.edu (J. W. Y. Choon), chong.chunwie@monash.edu (C. W. Chong), leesc@ha.org.hk (B. S. C. Lee), kenneth.lee@monash.edu (K. K. C. Lee)

${ }^{*}$ Corresponding author

\section{To cite this article:}

David Bin-Chia Wu, June Wai Yee Choon, Chun Wie Chong, Benjamin Shing Cheung Lee, Kenneth Kwing Chin Lee. Economic Evaluation of Ticagrelor in Treating Patients with Acute Coronary Syndrome in Hong Kong: A Cost-Utility Analysis. International Journal of Health Economics and Policy. Vol. 5, No. 3, 2020, pp. 63-71. doi: 10.11648/j.hep.20200503.13

Received: September 8, 2020; Accepted: October 6, 2020; Published: October 17, 2020

\begin{abstract}
This study aimed to evaluate the long-term cost-effectiveness of ticagrelor plus aspirin versus generic clopidogrel plus aspirin in acute coronary syndrome patients in Hong Kong (HK) from a public hospital's perspective. The study has adapted a previously developed two-component prediction model. The first component is a simple decision tree corresponding to the first year. Afterwards events in the second year onwards were estimated using a state-transition Markov model incorporating the potential of recurrent events such as myocardial infarction and strokes that could lead to death for estimating the long-term economic and health outcomes measured as cost per quality-adjusted life year (QALYs). Kaplan Meier survival analysis was employed to determine the risk of events. Probabilistic sensitivity analysis was used to estimate the probability of ticagrelor being cost-effective. A cost-effectiveness acceptability curve was used to estimate the willingness-to-pay of patients. The use of ticagrelor led to improved clinical outcomes by gaining additional life-years and QALYS over 5-year and lifetime time horizons. The incremental cost-effectiveness ratio was above 1 Gross Domestic Product (GDP) per capita only for the 1-year results. By replacing clopidogrel with ticagrelor for life-time, the incremental drug costs were offset by the substantial reduction in other direct costs, leading to an overall cost-savings of HK 2,878 per patient. The probabilistic sensitivity analysis showed that ticagrelor has $53.5 \%$ chance of being dominant and $34.7 \%$ being cost-effective at a threshold of 1 GDP per capita for Hong Kong. A cost-effectiveness acceptability curve also showed that the willingness-to-pay for ticagrelor was $90 \%$ at 1 GDP per capita. Ticagrelor plus aspirin appeared to be cost-effective over 5-year and life-time projection periods compared to clopidogrel plus aspirin.
\end{abstract}

Keywords: Ticagrelor, Acute Coronary Syndrome, Health Utility Analysis, Simulation Model, Cost Per QALY

\section{Introduction}

Since 1960s, cardiovascular diseases have been the third leading cause of death in Hong Kong [1]. Acute coronary syndrome (ACS) is a broad term with a spectrum of clinical presentations ranging from those for ST-segment elevation myocardial infarction (STEMI) to presentations found in nonST-segment elevation myocardial infarction (NSTEMI) or in unstable angina.
As per practice guidelines by the American College of Cardiology/American Heart Association Task Force, in patients with non-ST elevation acute coronary syndrome (NSTE-ACS), initiation of dual antiplatelet therapy of the combination aspirin and clopidogrel or aspirin and ticagrelor is recommended for up to 12 months [2]. In Hong Kong, similar guidelines are followed for the management of acute coronary syndrome. Ticagrelor is an oral antiplatelet drug indicated in patients with acute coronary syndrome to prevent 
thrombotic episodes.

The PLATelet inhibition and patient Outcomes (PLATO) trial was a multinational, multicenter, double-blind, double-dummy, randomized trial of 18,624 patients with ST-segment elevation or non-ST-segment elevation ACS [3, 4]. Patients were randomized to treatment with clopidogrel or ticagrelor after admission and within 24 hours of the acute event. The study found that in acute coronary syndrome patients initially intended for non-invasive management, ticagrelor was shown to have achieved a clinically important reduction in ischaemic events and mortality compared with clopidogrel without increasing major bleeding. In addition, the reduction of ischaemic events and mortality by ticagrelor compared to clopidogrel was consistent in acute coronary syndrome patients with non-ST elevation who did not actually have revascularization. In a subgroup analysis on Asian patients, there was no significant difference between Asian and non-Asian patients [5].

Based on findings from the PLATO trial, several studies have been conducted to assess the cost-effectiveness of treating ACS patients with ticagrelor compared to clopidogrel in Sweden, Germany, Canada, Thailand and Singapore [6-10]. Findings from all studies showed that a 12-month treatment with ticagrelor was more cost-effective compared to treatment with clopidogrel. However, cost effectiveness results across countries cannot be easily generalized due to differences in drug price, healthcare financing system, epidemiology and demographics. In the present study, we aimed to evaluate the short- and long-term cost-effectiveness of ticagrelor or clopidogrel plus aspirin in patients with ACS in the public sector of Hong Kong. The findings and the data generated therefrom would in turn assist policy-makers in their allocation of resources. The study was performed from the public payer's perspective.

\section{Main Body}

\subsection{Methodology}

\subsubsection{Model for First Year}

The present study has adapted a two-component prediction model (Figure 1) developed by Nikolic et al. [6]. The first component is a simple decision tree corresponding to the first year. Afterwards events in the second year onwards were estimated using a state-transition Markov model incorporating the potential of recurrent event such as myocardial infarction (MI) or stroke that could lead to death for estimation of the long-term economic and health outcomes measured as cost per quality-adjusted life year (QALYs).

Model inputs and the sources corresponding to the first year following treatment are shown in Table 1, and those for subsequent years are in Table 2 [6, 11-15]. In our analysis, both clinical outcomes and resource counts were based on data reported from the PLATO study as few local data was available at the time of this study. The total health-care costs per patient were used to estimate the mean per-patient health-care cost for each treatment group. However, the cost of rehabilitation was not included as part of resource use. The daily drug price was multiplied by the number of days patients were on the study drug. In order not to underestimate drug costs with ticagrelor in the cost-effectiveness analysis, the cost of study drugs was entered as a separate parameter and applied as long as patients remained alive during the 12 months of therapy. A cost of $75 \mathrm{mg}$ generic clopidogrel (HKD5.50 per day, lowest available price at the time of study) and $180 \mathrm{mg}$ ticagrelor (HKD22 per day) was applied. Patients' life expectancy was calculated using the Swedish Projected Life Tables, 2010 - 2039 [16].

\subsubsection{Markov Model for Long-Term Extrapolation}

Outcomes from the second year onwards were extrapolated with the Markov model, using results from the first year. Direct health care costs were partially HK-specific and utility data was adopted from published literature [17-21]. Time horizons were set at 1 year, 5 years and patients' lifetime.

To estimate long-term cost-effectiveness, the model was used to estimate number of quality-adjusted life-year (QALY) gained and cost of a non-fatal MI, a non-fatal stroke, or no MI or stroke which occurred during the 12 months of therapy. No treatment effect was incorporated in the model once patients were no longer on the study medications; therefore, the Markov model is identical for ticagrelor- and clopidogrel-treated patients.

For patients surviving and not suffering a non-fatal MI or stroke during the 12 months of therapy, the annual risks of non-fatal MI and non-fatal stroke (transitions 1 and 2 in Figure 1) were estimated by extrapolating out the observed hazard function of clopidogrel-treated patients in PLATO beyond 1 year of follow-up. The annual mortality risk (transition 3 in Figure 1) in the no-event state was estimated using age-specific mortality rates from Hong Kong life tables [22].

Similarly, survival after non-fatal events was modelled by estimating the health hazard ratio (HR) corresponding to the increased hazard of death following an MI or stroke relative to standard mortality rates from life tables. Different estimates were applied the first year after a non-fatal event [non-fatal MI state (transition 4 in Figure 1) and non-fatal stroke state (transition 5 in Figure 1)] when compared with the second year onwards [post-MI state (transition 6 in Figure 1) and post-stroke state (transition 7 in Figure 1)]. long-term costs, each state in the Markov model was assigned a cost estimate. This data is summarized in Table 2 for estimation.

With reference to the no-event state, PLATO data was analyzed to estimate the associated annual cost. The costs associated with a non-fatal event in the Markov model (non-fatal MI and non-fatal stroke states the first year, and the post-MI and post-stroke states the second year and onwards) were derived from the literature $[6,9,10]$.

Regarding long-term QALYs, the QALY estimate for patients without an event in the PLATO study was applied in the no-event state. The mean estimate of ticagrelor- and clopidogrel-treated patients was applied for patients aged 70 years. As patients grow older in the model, a proportional decrease due to age was applied [23]. 
For the non-fatal MI, non-fatal stroke, post-MI, and poststroke states, the decrements associated with the non-fatal MI and non-fatal stroke clinical pathways in the PLATO study were applied. The decrements were subtracted from the QALY estimate applied in the no-event state in the model. The QALY estimates for the long- term extrapolation are summarized in Table 3.

For subsequent years, mortality rates were based on HK-specific general population life tables, inflated by a hazard ratio (from PLATO and published references relevant to the nature of the state (no event, MI or stroke) and time since the event (first year or second and subsequent years) [11-13, 23].

\subsubsection{Outcome Assessment}

Health outcomes were estimated in terms of quality-adjusted life years (QALYs). The primary outcome measure was the cost per QALY gained. Therapy would be considered cost-effective if the incremental cost-effectiveness ratio (ICER) is below 1 Gross Domestic Product (GDP) per capita based on the recommendation of WHO (i.e. USD \$43,700), 1US\$=7.8HK\$) [24]. Costs are expressed in $\mathrm{HK} \$$ at 2016 prices. Both cost and effectiveness were discounted at $3 \%$ per annum.

\subsubsection{Event Risks, Costs and Quality of Life from the PLATO Study}

The risk of different clinical pathways, by treatment strategy, was estimated for the 12 months of therapy as reported in the PLATO study:

a. a non-fatal MI occurring before a potential non-fatal stroke with no subsequent fatal event.

b. a non-fatal stroke occurring before any potential non-fatal MI with no subsequent fatal event.

c. death occurring at any point in the study follow-up

d. no further event, which is one minus the combined risk of the other three clinical pathways.

Kaplan Meier survival analysis was employed to determine the risk of events, and the results of this analysis were incorporated into the model [25]. Then, a best-fit parametric time-to-event survival model with a Weibull distribution was selected in order to determine the baseline risk (the risk of clinical pathways in the clopidogrel group) and a hazard ratio (the treatment effect of ticagrelor) was applied to the baseline risk [26].

Survival models, i.e. Cox regression, were run to estimate different baseline event rates (clopidogrel group) associated with selected subgroups. As there was no statistically significant interaction for the primary endpoint between treatment and the final index hospitalization diagnosis $(\mathrm{P}>0.41)$, between treatment and medical history of diabetes mellitus $(\mathrm{P}>0.49)$, and between treatment and planned treatment approach $(\mathrm{P}>0.88)$, the HRs for the overall population were used to generate the event rates for ticagrelor-treated patients.

The importance of this assumption for the final results was investigated in alternative scenarios. The estimated risk of all-cause death for all ACS patients while on therapy was
0.046 and 0.059 for ticagrelor- and clopidogrel-treated patients, respectively. The corresponding risk of the MI clinical pathway was 0.050 and 0.058 for ticagrelor- and clopidogrel-treated patients, respectively. The risk of the stroke clinical pathway was 0.010 for ticagrelor-treated patients and 0.009 for clopidogrel-treated patients. The cost estimates for the 12 months of therapy were based on the resource use data from PLATO. Days on study drug, bed days due to hospitalizations, investigations, interventions, blood products and re-operations due to bleeding were recorded in the trial.

\subsubsection{Cost-Effectiveness Analysis}

Costs and QALYs were calculated over a lifetime time horizon and are presented as mean outcomes per patient. The estimated mean costs and QALYs were combined to calculate the incremental cost-effectiveness ratio (ICER) defined as: $I C E R=\frac{C_{T}-C_{C}}{E_{T}-E_{C}}$, where $\mathrm{C}$ is the estimated mean cost, $\mathrm{E}$ the estimated mean QALYs, and the treatment strategies are indexed $\mathrm{T}$ for ticagrelor and $\mathrm{C}$ for generic clopidogrel. A probabilistic sensitivity analysis (PSA) was performed by repeating 10,000 times the base case results and a cost-effectiveness acceptability curve was drawn to assess the probability of ticagrelor being more cost-effective than clopidogrel for increasing ICER values to assist making informed decision under different situations.

\subsection{Results}

\subsubsection{Base-Case Analysis}

The base-case results are presented in Table 3. Over a lifetime horizon, the use of ticagrelor led to improved clinical outcomes with a discounted survival of 13.08 QALYs per patient in the ticagrelor treatment group compared to 12.91 in the clopidogrel treatment group. This led to a gain of 0.17 QALY per patient (or a gain of 0.21 life year without considering patient's quality-of-life). By replacing generic clopidogrel, despite its higher daily cost of HKD22 (USD2.8, $1 \mathrm{USD}=7.8 \mathrm{HKD}$ ), ticagrelor was predicted to reduce direct medical treatment cost by HKD2,878 (USD369, $1 \mathrm{USD}=7.8 \mathrm{HKD}$ ) per patient over life-time. Over a 5-year horizon, the use of ticagrelor also led to better clinical outcomes by producing a discounted survival of 3.87 QALYs per patient in the ticagrelor treatment group compared to 3.82 in the clopidogrel treatment group. This led to a gain of 0.046 QALY per patient (a gain of 0.051 life year without considering patient's quality-of-life). Yet ticagrelor is expected to slightly increase direct medical treatment cost by HKD288 (USD37, 1USD=7.8HKD) per QALY over 5 years. Over 1-year time horizon, replacing generic clopidogrel by ticagrelor could generate a discounted survival of 0.8399 QALYs per patient in the ticagrelor treatment group compared to 0.8348 in the clopidogrel treatment group, leading to 0.0051 QALY gained (or 0.0062 life year without considering patient's quality-of-life). Ticagrelor was expected to incur an increase of direct medical treatment cost by HKD2,490 (USD319, 1USD=7.8HKD) per patient in 1 year. ICER values when clopidogrel was replaced by ticagrelor for 
long term were negative suggestive of ticagrelor being dominant mainly driven by its long-term cost savings. ICER values for 5 years were below the national threshold for Hong Kong whereas ICER values for 1 year were above the national threshold suggesting it may not be cost-effective.

\subsubsection{One-Way Sensitivity Analysis}

The sensitivity analyses indicated that the most influential parameters to ICER were cost of no event within trial (ticagrelor arm), cost of no event within trial (clopidogrel arm), probability of MI within trial (clopidogrel arm), and probability of MI within trial (ticagrelor arm) (Figure 2). The cost-effectiveness results of the base-case analysis were robust to plausible changes in the remaining input parameters.

\subsubsection{Probabilistic Sensitivity Analysis (PSA)}

Results of PSA showed that $53.5 \%$ of the plots were in the lower right quadrant suggesting that ticagrelor was dominant over clopidogrel and a total of $88 \%$ of all plots fell below the 1 GDP threshold of HK336,500 suggesting ticagrelor had a high probability to be cost-effective (Figure 3 ).

\subsubsection{Cost-Effectiveness Acceptability Curve}

Using HK336,500 (1 GDP for Hong Kong in 2016) as the acceptability threshold, it was found that the probability of ticagrelor being cost-effective was $90 \%$ (Figure 4 ).

\subsection{Discussion}

The current study adopted a previously published economic prediction model for the estimation of the shortand long-term cost effectiveness of ticagrelor plus aspirin versus clopidogrel plus aspirin which is the most commonly used treatment for patients with ACS. Although the same model was used in a number of similar studies in both western and Asian countries which have demonstrated the long term economic benefits by replacing clopidogrel with ticagrelor, the results of the current study offer new information in several areas. First, the current study examined the cost-effectiveness of ticagrelor over a short and medium term ( 1 and 5 years) as well as lifetime horizon whereas the previous Asian studies mainly focused on short term and life-time impacts. Our approach therefore provides a better insight on spending of limited resources in a wiser and evidence-based manner. Secondly, the study that was carried out in Singapore was performed from the perspective of only one general public healthcare institution whereas the study perspective of the current study was specifically from the Hospital Authority of Hong Kong, the single largest public healthcare provider in the region. Hospital Authority manages 43 public hospitals and institutions, 49 specialist outpatient clinics and 73 general outpatient clinics [27]. In addition, the healthcare financing system is unique for every jurisdiction. Therefore, findings from the present study offers new knowledge to the current pool for other countries in Asia with a single-payer healthcare system such as Malaysia and Indonesia. Thirdly, the findings of the present study are different in terms of cost-effectiveness of ticagrelor from those of other previous studies as our results suggested that ticagrelor may not be cost effective in short term use but will be cost effective if used in medium and long term.

Antiplatelet agents represent one of the high-spending items in the government's annual healthcare budget. Hence, results from this study have high significance in terms of healthcare budget consideration because the conventional way of determining funding decision has always been mainly based on clinical outcomes and very little consideration is given to economic impact on a long-term basis. The results of the present study suggest that ticagrelor, as measured by \$/QALY gained, is cost-effective both in medium and long-term according to the WHO proposed standard despite its much higher initial acquisition cost than clopidogrel.

This study also demonstrated that an economic prediction model developed in overseas countries can be adopted in local setting after some modifications in the early screening process. The model should also be populated with as much local data as possible in order to generate results that are reflective of the local settings. The model used in this study was also used in earlier studies in Singapore and Thailand, results from these and the current were fairly consistent but only the current study showed that potential savings was possible in the long run.

When clopidogrel was replaced by ticagrelor, the economic impact due to ticagrelor as measured by ICER is likely to be cost-effective over 5 years and cost savings was possible in long term estimation. It was a classic example in which disease management using a new drug may be initially more expensive due to high unit cost but may end up in cost savings in the long run due to higher efficacy and/or better safety profile and eventually a reduction in the cost of management. It further implied that decision making should not be made solely on the initial unit drug cost because many new drugs can potentially lead to long-term healthcare savings due to improved therapeutic effect and reduced adverse reactions.

The one-way sensitivity analysis results suggest that the ICER value was sensitive to occurrence of event and probability of myocardial infarction within trial for both the ticagrelor and clopidogrel arms. The implication of this finding is important as the superiority of ticagrelor being more cost-effective seems to be related to the cardiac status of the patient. Hence using ticagrelor in patients who are more stable are more likely to end up with cost-effective outcomes.

\subsubsection{Limitations of the Study}

There are a few limitations in the present study that would warrant careful interpretation of our study results. These include:

1) The efficacy of ticagrelor in preventing more deaths compared to clopidogrel was extrapolated beyond the 1-year PLATO trial period due to a lack of local relevant data. Hence further relevant evidence as time progresses is required. It is thus important to perform some post-market trials or observational studies after ticagrelor has become widely used after its launch to 
substantiate the current claims.

2) In applying the model, it was assumed that ACS patients in Hong Kong were treated in a similar manner to those in PLATO study. However, this assumption could also lead to potential bias and over estimation of cost because randomized clinical trials are mostly protocol-driven and hence patients enrolled in trials tend to receive closer monitoring and hence compliance is better when compared to those in real world clinical setting.

3) The cost of clopidogrel used in the current study may change as a number of generic forms of clopidogrel is supplied by the public hospitals through tender arrangements in Hong Kong. The current cost was only a median cost of the available generics. Nevertheless, due to the big difference in unit cost between ticagrelor and generic clopidogrel, it is anticipated that the overall conclusion of the study will remain unchanged. Moreover, our one-way sensitivity analysis results did not show cost of clopidogrel to be one of the most sensitive parameters.

\subsubsection{List of Abbreviations}

1) ACS: acute coronary syndrome

2) GDP: Gross Domestic Product

3) HK: Hong Kong

4) ICER: incremental cost-effectiveness ratio

5) MI: myocardial infarction

\subsection{Figure}
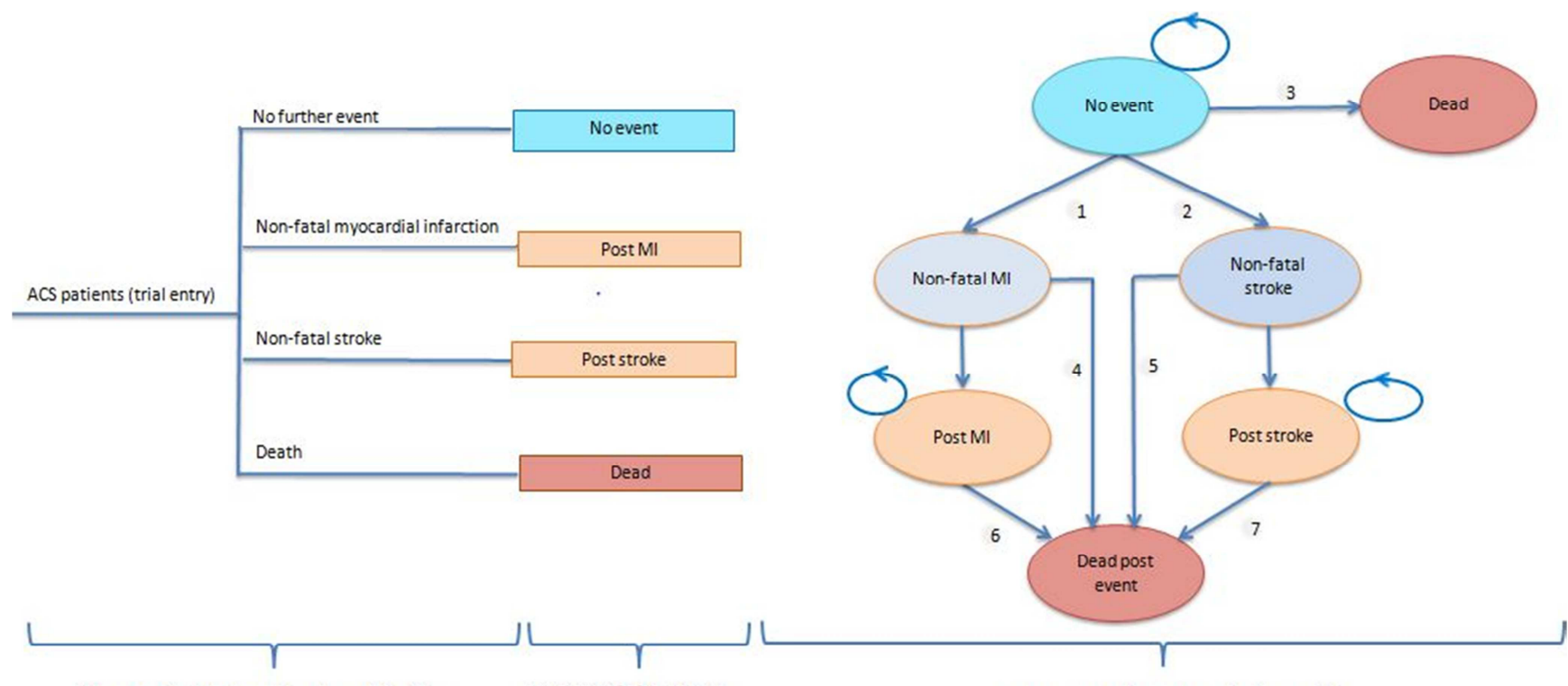

One-year decision tree based on plato data

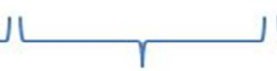

Start state in long-term Markov model
6) NSTE-ACS: non-ST elevation acute coronary syndrome

7) NSTEMI: non-ST-segment elevation myocardial infarction

8) PLATO: PLATelet inhibition and patient Outcomes (PLATO) trial

9) PSA: probabilistic sensitivity analysis

10) QALY: quality-adjusted life year

11) STEMI: ST-segment elevation myocardial infarction

12) WHO: World Health Organization

\section{Conclusion}

The present study has generated evidence based on health economic assessment that the treatment of ACS patients with lifetime use of ticagrelor plus aspirin can potentially reduce the cost of management and increase the efficiency due to better health outcomes as compared with generic clopidogrel plus aspirin. Our projection model shows that ticagrelor therapy appears to be cost-saving both on medium- and long-term assessment in the public healthcare sector of Hong Kong. Hence it is important for both clinicians and budget decision makers to include long term benefits in their consideration instead of focusing on the drug's immediate budget impact. Our study results may also be generalizable to other jurisdictions with a similar healthcare funding model.

Figure 1. Model Structure.

Markov model transitions in figure: (1) risk of non-fatal myocardial infarction for patients with no myocardial infarction (MI) or stroke in the PLATO study. (2) Risk of non-fatal stroke for patients with no MI or stroke in the PLATO study. (3) Mortality risk for patients with no MI or stroke in the PLATO study. (4) Mortality risk at the first year after a non-fatal myocardial infarction. (5) Mortality risk at the first year after a non-fatal stroke. (6) Mortality risk at second and subsequent years after a non-fatal myocardial infarction. (7) Mortality risk at second and subsequent years after a non-fatal stroke. 
Cost of no event within trial (ticagrelor arm) Cost of no event within trial (clopidogrel arm) Probability of MI within trial (clopidogrel arm) Probability of MI within trial (ticagrelor arm) Probability of death within trial (dopidogrel arm) Cost of MI within trial (ticagrelor arm) Daily cost of ticagrelor Utility of no event within trial (clopidogrel arm) Utility of no event within trial (ticagrelor arm) Cost of death within trial (ticagrelor arm) Discount Rate, outcome Cost of death within trial (clopidogrel arm) Discount Rate, cost Daily cost of clopidogrel Cost of MI within trial (clopidogrel arm) Cost of stroke within trial (ticagrelor arm) Utility MI within trial (ticagrelor arm) Cost of stroke within trial (clopidogrel arm) Probability of stroke within trial (ticagrelor arm) Utility MI within trial (clopidogrel arm) Probability of stroke within trial (clopidogrel arm) Utility stroke within trial (ticagrelor arm) Utility stroke within trial (clopidogrel arm)

\begin{tabular}{llllll}
\hline$-200,000$ & $-150,000$ & $-100,000$ & $-50,000$ & 0 & 1 \\
& & Cost per QALY gained (HKS 2014) & 50,000 & 100,000
\end{tabular}

Figure 2. One-way Sensitivity Analysis.

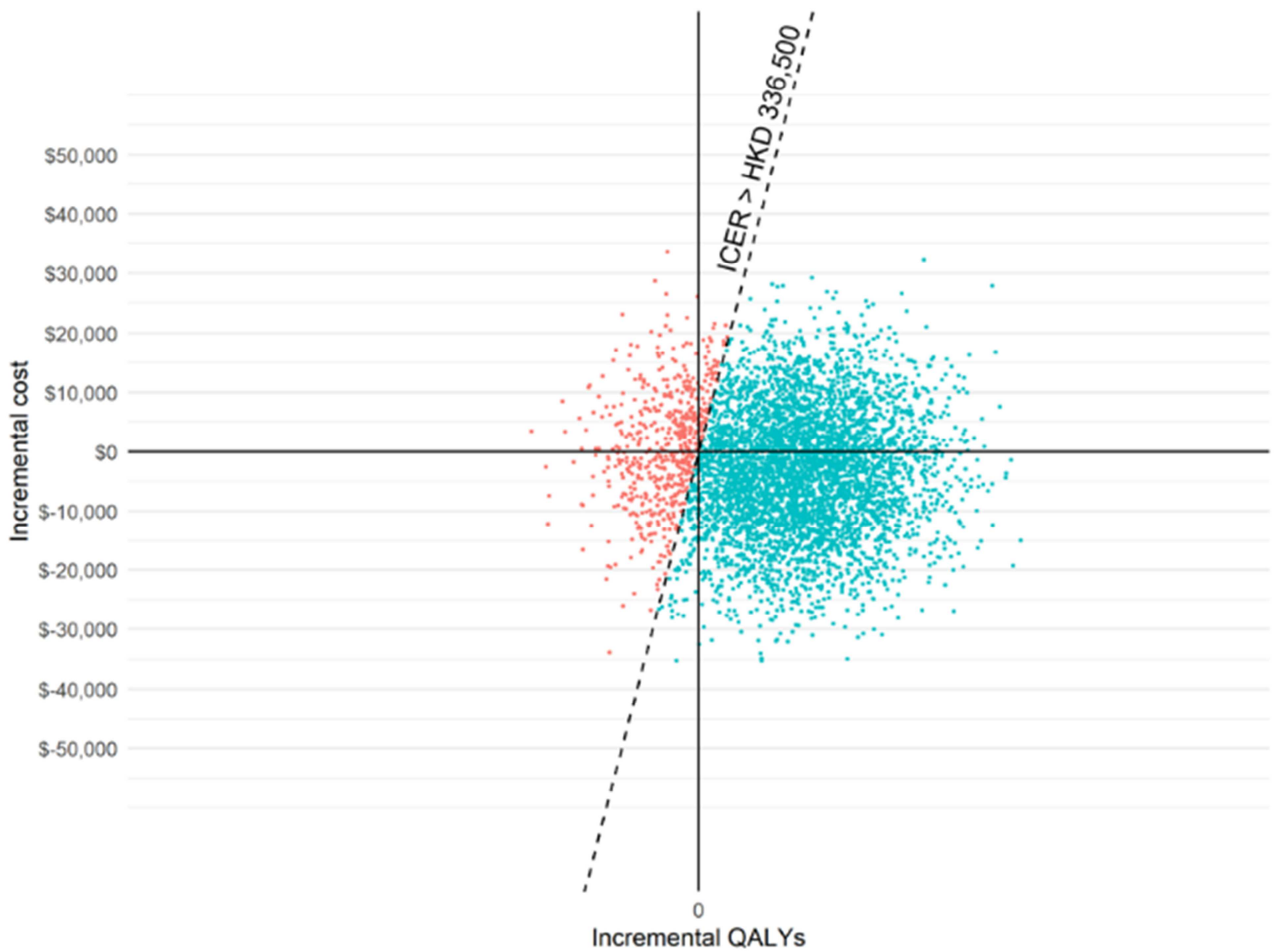

Figure 3. Probabilistic Sensitivity Analysis. 


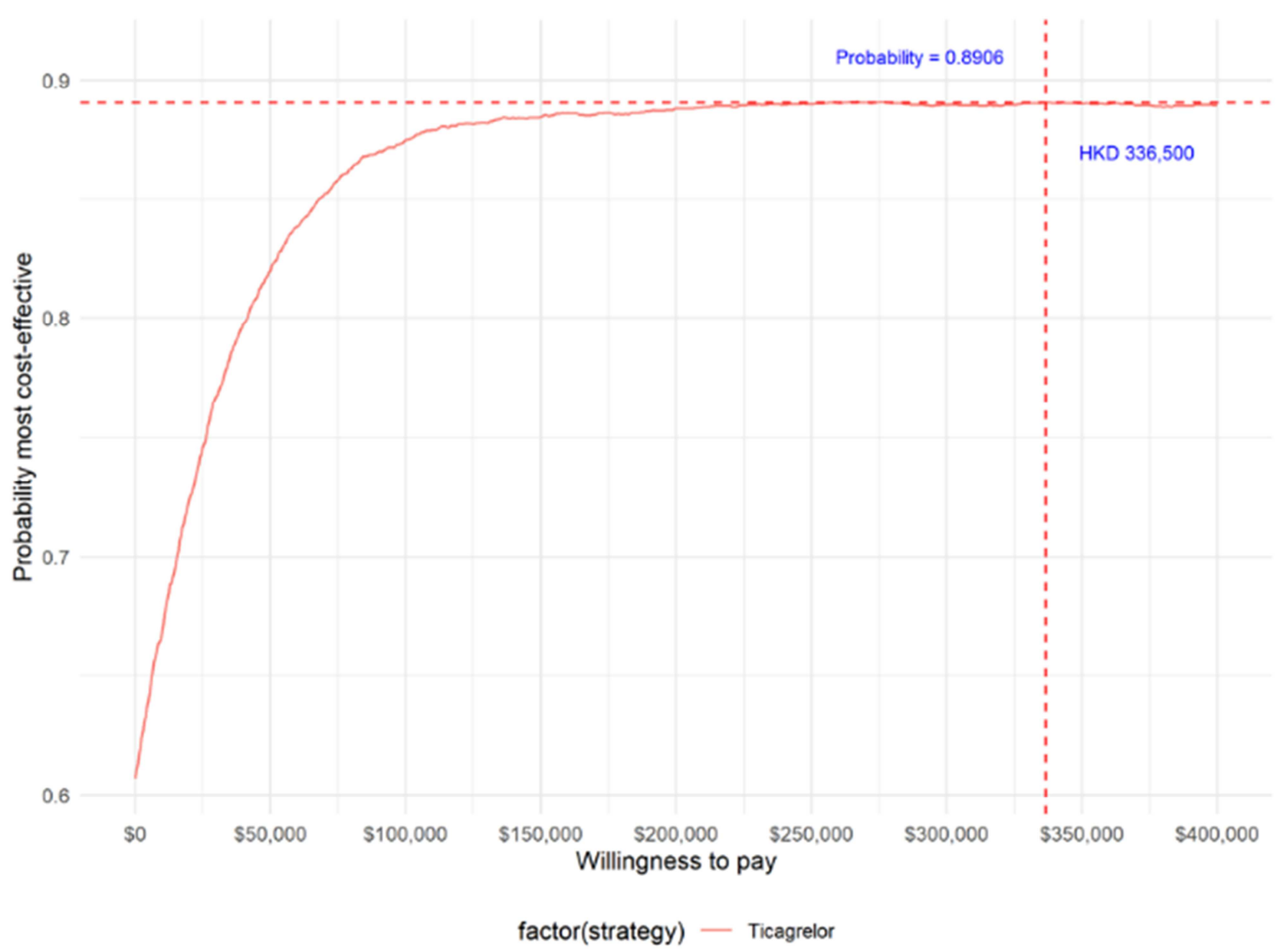

Figure 4. Cost-Effectiveness Acceptability Curve.

\subsection{Table}

Table 1. Data inputs for one-year decision tree model.

\begin{tabular}{|c|c|c|c|}
\hline Parameters & Ticagrelor & Clopidogrel & Source \\
\hline \multicolumn{4}{|l|}{ Probability } \\
\hline All-cause death & 0.0462 & 0.0586 & PLATO data $[4,5]$ \\
\hline Nonfatal MI & 0.0497 & 0.0575 & PLATO data $[4,5]$ \\
\hline Nonfatal Stroke & 0.0096 & 0.0088 & PLATO data $[4,5]$ \\
\hline No event & 0.8945 & 0.8751 & PLATO data $[4,5]$ \\
\hline \multicolumn{4}{|l|}{ Cost (HKS) } \\
\hline All-cause death & 114,102 & 115,587 & Nickolic et al [6], Chin [10] \\
\hline MI & 158,186 & 160,671 & Nickolic et al [6], Chin [10] \\
\hline Stroke & 136,269 & 138,760 & Nickolic et al [6], Chin [10] \\
\hline No event & 83875 & 86366 & Nickolic et al [6], Chin [10] \\
\hline \multicolumn{4}{|c|}{ Daily cost of $180 \mathrm{mg}$} \\
\hline Ticagrelor & 22.0 & N/A & Current market price \\
\hline \multicolumn{4}{|c|}{ Daily cost of $75 \mathrm{mg}$} \\
\hline Clopidogrel & N/A & 5.5 & Current market price \\
\hline \multicolumn{4}{|l|}{$Q A L Y$} \\
\hline All-cause death & 0.2473 & 0.2503 & PLATO data $[4,5]$ \\
\hline MI & 0.8106 & 0.8136 & PLATO data $[4,5]$ \\
\hline Stroke & 0.7349 & 0.7379 & PLATO data $[4,5]$ \\
\hline No event & 0.8732 & 0.8763 & PLATO data $[4,5]$ \\
\hline
\end{tabular}


David Bin-Chia Wu et al: $\quad$ Economic Evaluation of Ticagrelor in Treating Patients with Acute

Coronary Syndrome in Hong Kong: A Cost-Utility Analysis

Table 2. Data inputs for Markov model for long-term extrapolations.

\begin{tabular}{llll}
\hline Epidemiological parameters & Base-case value & Range for SA & Source \\
\hline Annual risk of MI in the no event state & 0.019 & $0-6 \%$ & PLATO data [4, 5] \\
Annual risk of stroke in the no event state & 0.003 & $\pm 25 \%$ & PLATO data [4, 5] \\
Increased risk of death in the no event state & 2 & $\pm 25 \%$ & Statistics Sweden [20] \\
Increased risk of death in the non-fatal MI state & 6 & $\pm 25 \%$ & PLATO data [4, 5] \\
Increased risk of death in the post-MI state & 3 & $\pm 25 \%$ & Assumption \\
Increased risk of death in the non-fatal stroke state & 7.43 & $\pm 25 \%$ & Dennis et al. [11] \\
Increased risk of death in the post-stroke state & 3 & $\pm 25 \%$ & Dennis et al. and Olai et al. [11, 12] \\
Cost parameters & & & \\
Annual cost in the non-fatal MI state (E) & 94,886 & $\pm 50 \%$ & Vivian W. Lee [18] \\
Annual cost in the post-MI state (E) & 86,710 & $\pm 50 \%$ & Vivian W. Lee [18] \\
Annual cost in the non-fatal stroke state (E) & 101,998 & $\pm 50 \%$ & Elsie Hui [19] \\
Annual cost in the post-stroke state (E) & 10,305 & $\pm 50 \%$ & Elsie Hui [19] \\
Annual cost in the no event state (E) & 0 & 0 & Assumption \\
Utility parameters & & & \\
Annual QALY weight in the non-fatal MI state age, 69 & 0.8748 & $\pm 10 \%$ & PLATO data [4, 5] \\
Annual QALY weight in the non-fatal MI state age 70-79 & 0.8430 & $\pm 10 \%$ & Burstrom and Rehnberg [21] \\
Annual QALY weight in the non-fatal MI state age. 79 & 0.7814 & $\pm 10 \%$ & Burstrom and Rehnberg [21] \\
Annual QALY decrement non-fatal MI state & 0.0627 & $\pm 10 \%$ & PLATO data [4, 5] \\
Annual QALY decrement post-MI state & 0.0627 & $\pm 10 \%$ & PLATO data [4,5] \\
Annual QALY decrement non-fatal stroke state & 0.1384 & $\pm 10 \%$ & PLATO data [4,5] \\
Annual QALY decrement post-stroke state & 0.1384 & $\pm 10 \%$ & PLATO data [4,5] \\
\hline
\end{tabular}

Table 3. Base-case results.

\begin{tabular}{lllll}
\hline & Ticagrelor & Clopidogrel & Incremental & ICER \\
\hline Lifetime & & & & Baseline \\
Costs & 493,022 & 499,283 & $-6,261$ & Cost-saving \\
Life-years & 15.8444 & 15.6380 & 0.2064 & Cost-saving \\
QALYs & 13.0881 & 12.9135 & 0.1746 & Baseline \\
5 years & & & Cost-saving \\
Costs & 250,114 & 253,209 & $-3,096$ & Cost-saving \\
Life-years & 4.4771 & 4.4256 & 0.0515 & Baseline \\
QALYs & 3.8752 & 3.8292 & 0.0460 & Cost-saving \\
1 year & & & -893 & Cost-saving \\
Costs & 222,227 & 223,120 & 0.0062 & 0.0051 \\
Life-years & 0.9769 & 0.9707 & & \\
QALYs & 0.8399 & 0.8348 & & \\
\hline
\end{tabular}

\section{References}

[1] Department of Health The government of the Hong Kong Special Administrative Region: Coronary Heart Disease. Available from: http://www.healthyhk.gov.hk/phisweb/en/healthy_facts/diseas e_burden/major_causes_death/coronary_heart_disease/. Accessed 28 September 2018.

[2] Amsterdam EA, Wenger NK, Brindis RG, et al. 2014 AHA/ACC Guideline for the Management of Patients With Non-ST-Elevation Acute Coronary Syndromes: A Report of the American College of Cardiology/American Heart Association Task Force on Practice Guidelines. J Am Coll Cardiol. 2014; 64 (24): e139-e228.
[3] Wallentin L, Becker RC, Budaj A, Cannon CP, Emanuelsson H, Held C, et al. Ticagrelor versus clopidogrel in patients with acute coronary syndromes. N Engl J Med. 2009; 361: 1045-57.

[4] James SK, Roe MT, Cannon CP, et al. Ticagrelor versus clopidogrel in patients with acute coronary syndromes intended for non-invasive management: substudy from prospective randomised PLATelet inhibition and patient Outcomes (PLATO) trial. BMJ. 2011; 342: d3527.

[5] Kang HJ, Clare RM, Gao R, Held C, Himmelmann A, James SK, Lim ST, Santoso A, Yu CM, Wallentin L, Becker RC; PLATO Investigators. Ticagrelor versus clopidogrel in Asian patients with acute coronary syndrome: A retrospective analysis from the Platelet Inhibition and Patient Outcomes (PLATO) Trial. Am Heart J. 2015 Jun; 169 (6): 899-905. e1. doi: 10.1016/j.ahj.2015.03.015. 
[6] Nikolic E, Janzon M, Hauch O, et al. Cost-effectiveness of treating acute coronary syndrome patients with ticagrelor for 12 months: results from the PLATO study. Eur Heart J. 2013; 34: $220-8$

[7] Theidel U, Asseburg C, Giannitsis E, et al. Cost-effectiveness of ticagrelor versus clopidogrel for the prevention of atherothrombotic events in adult patients with acute coronary syndrome in Germany. Clin Res Cardiol. 2013; 102: 447-58.

[8] Grima DT, Brown ST, Kamboj L, et al. Cost-effectiveness of ticagrelor versus clopidogrel in patients with acute coronary syndromes in Canada. Clinicoecon Outcomes Res. 2014; 6: 49-62.

[9] Yamwong S, Permsuwan U, Tinmanee S, et al. Long-term cost effectiveness of ticagrelor in patients with acute coronary syndromes in Thailand. Health Economics Review. 2014; 4: 1-7.10.

[10] Chin CT, Mellstrom C, Chua TS, et al. Lifetime cost-effectiveness analysis of ticagrelor in patients with acute coronary syndromes based on the PLATO trial: a Singapore healthcare perspective. Singapore Med J. 2013; 54: 169-75.

[11] Dennis MS, Burn JP, Sandercock PA, et al. Long term survival after first-ever stroke: the Oxfordshire Community Stroke Project. Stroke. 1993; 24: 796-800.

[12] Olai L, Omne-Ponten M, Borgquist L, et al. Survival, hazard function for a new event, and healthcare utilization among stroke patients over 65 years old. Stroke. 2009; 40: 3585-90.

[13] Ministry of Health Singapore: Hospital Bill Sizes. 2013. http://www.moh.gov.sg/content/moh_web/home/costs_and_fin ancing/HospitalBillSize.html. Accessed 16 Sept 2018.

[14] Management Center for Health Promotion, Republic of Korea. Cost- effectiveness analysis of national prevention programs for cardiovascular disease. 2007.

[15] Census and Statistics Department. Hong Kong life tables 1971-2018.

http://www.censtatd.gov.hk/hkstat/sub/sp190.jsp?productCode $=$ D5320184. Accessed 20 August 2018 .

[16] Burström K, Johannesson M, Rehnberg C. Deteriorating health status in Stockholm 1998-2002: results from repeated population surveys using the EQ-5D. Qual Life Res. 2007; 16: 1547-53.
[17] Census and Statistics Department. Hong Kong Population Projections 2010-2039. Available at: http://www.statistics.gov.hk/pub/B1120015042010XXXXB01 00.pdf. 2010. Accessed 1 Sept 2018.

[18] Lee VWY, Chan WK, Lam NLC, et al. Cost of acute myocardial infarction in Hong Kong. Dis Manage Health Outcomes. 2005; 13: 281-5.

[19] Hui E, Lum CM, Woo J, et al. Outcomes of elderly stroke patients day hospital versus conventional medical management. Stroke. 1995; 26: 1616-19.

[20] Statistics Sweden. Life Tables 2005-2009 for men and women. 2017. http://www.scb.se. Accessed 23 Sept 2018.

[21] Burström K, Rehnberg C. Health-related quality of life in Stockholm County 2002. Results from repeated population surveys using the EQ-5D. 2007. https://www.researchgate.net/publication/6011225_Deteriorati ng health status in Stockholm 1998-2002 Results from re peated_population_surveys_using_the_EQ-5D. Accessed 1 Oct 2018

[22] Census and Statistics Department. https://www. censtatd.gov.hk/hkstat/hkif/index.jsp. 2018 Accessed 20 Sept 2018.

[23] Census and Statistics Department. The Government of the Hong Kong Special Adminsitrative Region. 2018. http:/ www.censtatd.gov.hk/hkstat/sub/bbs.jsp. Accessed 30 Sept 2018.

[24] Choosing interventions that are cost-effective. Geneva: World Health Organization. 2014. http://www.who.int/choice/en/Accessed28Sept2018.

[25] Collett D. Modelling Survival Data. In: Medical Research. 2nd ed. London: Chapman and Hall/CRC; 2003.

[26] Briggs AH, Claxton K, Sculpher MJ. In: Decision Modelling for Health Economic Evaluation. Oxford: Oxford University Press; 2006.

[27] https://www.ha.org.hk/visitor/ha_visitor_index.asp?Content_I $\mathrm{D}=10036 \&$ Lang $=$ ENG\&Ver=HTML2018. Accessed 28 Sept 2019. 